\title{
Depilatory creams increase the number of hair follicles, and dermal fibroblasts expressing interleukin-6, tumor necrosis factor- $\alpha$, and tumor necrosis factor- $\beta$ in mouse skin
}

\author{
Pi-Fen Tsai ${ }^{1, *}$, Fen-Pi Chou ${ }^{2, \#}$, Ting-Shuan Yu ${ }^{1}$, Huei-Jane Lee ${ }^{2,3, *}$, and Chun-Tang Chiu ${ }^{4, *}$ \\ 'Institute of Medicine, Chung Shan Medical University, Taichung 40221, ${ }^{2}$ Department of Biochemistry, School of Medicine, College of Medicine, Chung Shan \\ Medical University, Taichung 40221, ${ }^{3}$ Department of Clinical Biochemistry, Chung Shan Medical University Hospital, Taichung 40221, ${ }^{4}$ Department of Cosmetic \\ Science, Chang Gung University of Science and Technology, Taoyuan 33382, Taiwan
}

\author{
ARTICLE INFO \\ Received January 23, 2021 \\ Revised March 2, 2021 \\ Accepted April 5, 2021 \\ *Correspondence \\ Huei-Jane Lee \\ E-mail: Ihj@csmu.edu.tw \\ Chun-Tang Chiu \\ E-mail:nydrskin@yahoo.com.tw
}

Key Words

Dermis

Hair follicle

Inflammation

Interleukin-6

Tumor necrosis factor- $\alpha$

\#These authors contributed equally to this work.
ABSTRACT Besides using for hair removal, depilatory agents have been considered to be used as a penetration enhancer for transepidermal drug delivery. To examine the effect in hair follicles (HFs), two commercially available depilatory creams were tested on the dorsal skin of mice to monitor the effect deep into the skin structure. Fifteen male BALB/c mice were used in this study. Depilatory creams were applied to the dorsal skin of the same animal using shaved and untouched treatments as controls to minimize individual differences. Skin samples were collected at three days, one week and two weeks ( $n=5$ for each) after the treatment, and subjected for hematoxylin-eosin staining, and immunohistochemical analysis for proinflammatory cytokines. The morphological examination showed an increase in the thickness of epidermal layer of the depilatory cream-treated skin at early time points and in the subcutis at two weeks. Depilatory cream promoted entry of anagen phase and increased the number of hair follicles in the subcutis at one and two weeks. Immunohistochemistry showed elevated percentages of dermal fibroblasts expressing interleukin-6, tumor necrosis factor- $\alpha$, and tumor necrosis factor- $\beta$. Shaving process increased the thickness of epidermis and dermis as depilatory creams did, but did neither induce the expression of proinflammatory cytokines in the dermal fibroblasts nor the number of HFs. The results suggested that the commercially available depilatory creams caused a transient minor inflammatory response of the skin and increased the levels of cytokines that might subsequently affect hair growth.

\section{INTRODUCTION}

Chemical hair-removal products, also called depilatories, are cosmetic preparations used to remove hair from the skin. They work by breaking down the sulphur bonds in the hair's keratin that are sensitive to strong alkaline and deoxidation agents. The active ingredient for deoxidizing the sulphur bonds is a salt of thioglycolic acid. In order to attain a high concentration of hydroxide ions for keeping thioglycolic acid as an active dianion in the product, strong alkalis are used, such as sodium hydroxide [1].

Thioglycolic acid is a harsh chemical, and its high $\mathrm{pH}$ value means it can be harmful to the skin. Chemical depilation generally does not destroy the dermal papilla, and hair is able to grow back afterwards because relatively small amounts of chemicals are used [2]. The active ingredients are administered in a cream base containing numerous other substances such as emollients, emulsifiers, moisturizers, antioxidants, and plant extracts, to minimize side effects. Depilation is followed by careful rinsing (i) (8) This is an Open Access article distributed under the terms of the Creative Commons Attribution Non-Commercial License, which permits unrestricted non-commercial use, distribution, and reproduction in any medium, provided the original work is properly cited. Copyright $\odot$ Korean J Physiol Pharmacol, pISSN 1226-4512, elSSN 2093-3827
Author contributions: P.F.T. and F.P.C. performed most of the experiments, analyzed the data and wrote the draft. T.S.Y. conducted the animal experiment, collected the specimens and helped with data analysis. H.J.L. and C.T.C. contributed to study conception, experimental design and manuscript writing. All authors have approved the final manuscript for publication and agreed to be accountable for all aspects of the work. 
with water, and various conditioners are applied to restore the skin's $\mathrm{pH}$ to normal.

Depilatory agents have become the focus of clinical interest because some substances, such as theophylline and insulin, can enhance transdermal penetration in rats, suggesting that these substances can be used to improve transepidermal drug delivery [3-6]. The penetration-enhancing effects persist for 1 day, indicating that the structural changes caused by depilatory agents do not subside within 1 day [4]. Several ex vivo experiments have demonstrated that the intracellular and intercellular structures of stratum corneum of human skin are detected after treatment with a hair removal cream [6]. Experiments have also revealed that thioglycolate damages cornified envelopes isolated from the stratum corneum of the epidermis [7]. These observations suggest that the effects of hair removal cream on the surface of skin could be greater than expected.

Hair removal creams have been considered for use as penetration enhancers for transepidermal drug delivery because of their active ingredient, thioglycolate. The long-term effects of thioglycolate on the structure of the skin must be identified, as must the short-term effects on the epidermis. In this study, two brands of commercially available depilatory creams with calcium thioglycolate base were tested on the dorsal skin of mice to examine the skin's short-term (3 days and 1 week) and long-term ( 2 weeks) responses. The results revealed that the hair removal creams caused an increase number in hair follicles (HFs) and higher levels of interleukin-6 (IL-6), tumor necrosis factor- $\alpha$ (TNF- $\alpha$ ), and tumor necrosis factor- $\beta$ (TNF- $\beta$ ) in dermal fibroblasts.

\section{METHODS}

\section{Materials}

UltraVision Quanto Detection System HRP and DAB Quanto Chromogen and Substrate were purchased from Thermo Fisher Scientific (Waltham, MA, USA). Hydrogen peroxide (30\% w/v) was purchased from PanReac AppliChem (Darmstadt, Germany). Xylene, ethanol and hematoxylin were obtained from Muto pure chemicals (Tokyo, Japan). Antibodies used in this study were as follows: anti-IL- 6 and anti-TNF- $\alpha$ (Abcam, Cambridge, MA, USA), anti-TNF- $\beta$ (Affinity, Zhenjiang City, China). Two brands of depilatory creams were obtained from a local supermarket, and were marked as DC-N and DC-S in the text and figures.

\section{Experimental animals}

Male BALB/c mice, five weeks old, were obtained from the National Laboratory Animal Center (National Science Council, Taipei City, Taiwan). The use of mice was reviewed and approved by Chung Shan Medical University Animal Care Committee. Mice were housed in a temperature-controlled room $\left(22 \pm 2^{\circ} \mathrm{C}\right)$ and illuminated for $12 \mathrm{~h}$ daily at a humidity of $55 \pm 2 \%$. After one week of adaptation to the environment, experiments were carried out using six-week-old mice, since six- to nine-week-old mice were shown to be in the telogen stage of hair cycle [8]. The dorsal side of the animal $(n=5)$ was divided into four areas receiving the treatments as shown in Fig. 1P with one area untouched, one area shaved and two areas depilated by two brands of depilatory creams following the directions suggested by the manufacturers. At the indicated time points (three days, one week, and two weeks), mice were euthanized by carbon-dioxide asphyxiation followed by exsanguination. The skin samples were collected and used for further experiments.

\section{Hematoxylin-eosin staining}

Skin biopsies were fixed with $10 \%$ formalin, paraffin-embedded, and processed for hematoxylin-eosin (H\&E) staining. To observe the histological change, sections were deparaffinized with xylene, hydrated in a descending series of graded ethanol, and stained with hematoxylin for two min, followed by rising in gentle running water for two min and eosin staining for five seconds. The sections were dehydrated by incubation in an ascending series of graded ethanol and xylene and then mounted.

\section{HFs and dermal fibroblasts counting}

The number of HFs and dermal fibroblasts of each treatment of one animal was manually counted from the digital images of photomicrographs that were taken from three representative areas of each skin tissue section at a fixed magnification of $100 \times$ and cropped in a fixed area with a width of $1.5 \mathrm{~mm}$. The levels of cytokines were expressed as percentages of immunohistochemical (IHC)-positive cells (brown color) to 100 counted dermal fibroblasts.

\section{IHC analysis}

IHC analysis was performed on formalin-fixed and paraffinembedded skin tissue. Sections $(3 \mu \mathrm{m})$ on coated slides were deparaffinized with xylene, rehydrated in a descending series of graded ethanol and then subjected to block the endogenous peroxidase activity with $0.6 \% \mathrm{H}_{2} \mathrm{O}_{2}$. The sections were incubated with diluted primary antibodies as indicated by the manufacturers at $37^{\circ} \mathrm{C}$ for one hour, followed by detecting with UltraVision Quanto Detection System HRP and DAB Quanto Chromogen and Substrate (Thermo Fisher Scientific), counter-staining with Mayer's hematoxylin and mounting in glycerin.

\section{Statistical methods}

All data were presented as mean \pm standard deviation. Since different treatment groups were obtained from the same animals, 


\section{Control}

DC-S

DC-N

Shaved

3 days
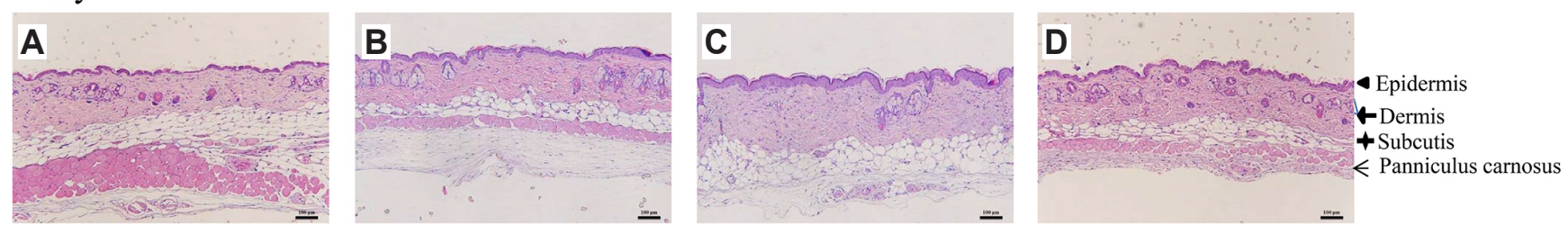

\section{1 week}
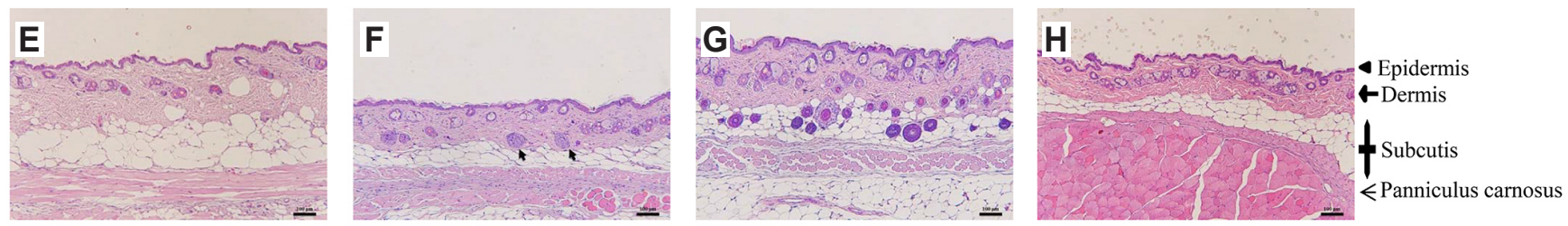

\section{2 weeks}
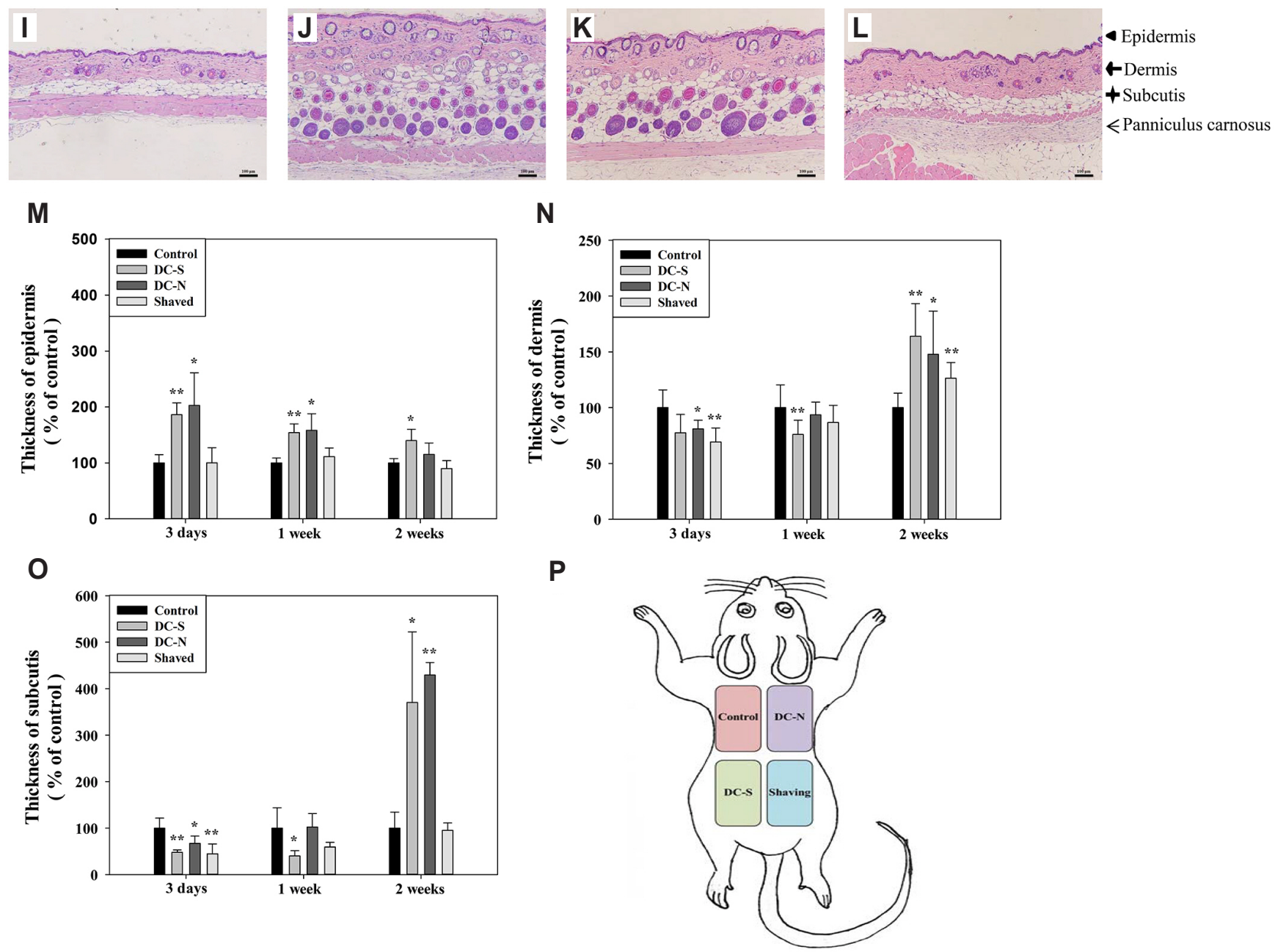

Fig. 1. Morphological changes of skin and subcutis induced by depilatory creams. Representative H\&E stain (10X) of transverse sections of dorsal skin tissues received topical application of depilatory creams, and shaved and control (untouched) treatments for three days (A-D), one week (E$H)$ and two weeks $(I-L)$ in BALB/C mice $(n=5)$. The thickness of epidermis $(M)$, dermis $(N)$ and subcutis $(O)$ layers of transverse skin sections received different treatments were measured and presented as percent of control. (P) Schematic diagram of application of two brands of depilatory creams (DC-N and DC-S) on mouse dorsal skin along with shaved and control treatments. All data were presented as mean \pm SD and analyzed by one-way RMANOVA with a post-hoc analysis of Holm Sidak test for pairwise comparisons. Arrow, hair follicle in the early anagen. ${ }^{*} p<0.05$ and ${ }^{* *} p<0.01$ vs. control. Scale bar, $100 \mu \mathrm{m}$. 
One-way repeated measures ANOVA (one-way RMANOVA) was used to test a significant effect with a post-hoc analysis of Holm Sidak test that was performed for pairwise comparisons to the untouched control (control). The $\mathrm{p}<0.05$ was considered statistically significant (Sigma-Stat 3.5, Jandel Scientific, San Rafael, CA, USA).

\section{RESULTS}

\section{Morphological changes of skin and subcutis induced by depilatory creams}

Since the presence of waves of hair growth in mouse skin [9], we have applied the tested materials to the same animal $(n=5)$ in order to eliminate the individual differences among animals. The activities of all animals were normal, and there were no signs of

\section{Control}

DC-S

DC-N

Shaved

3 days
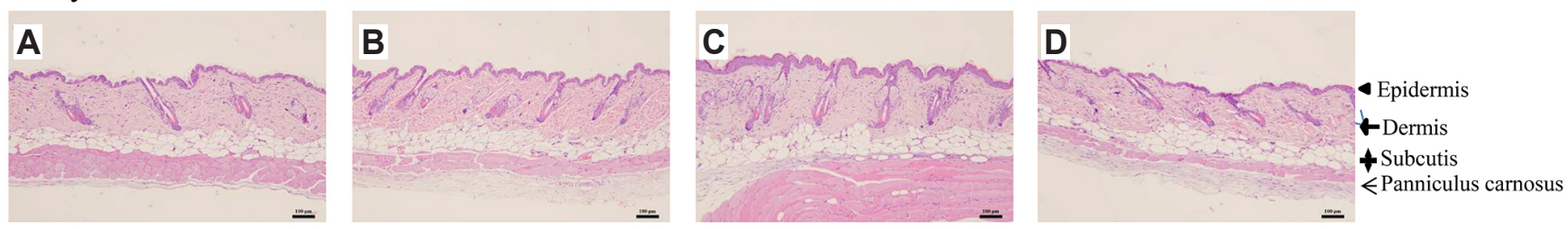

1 week
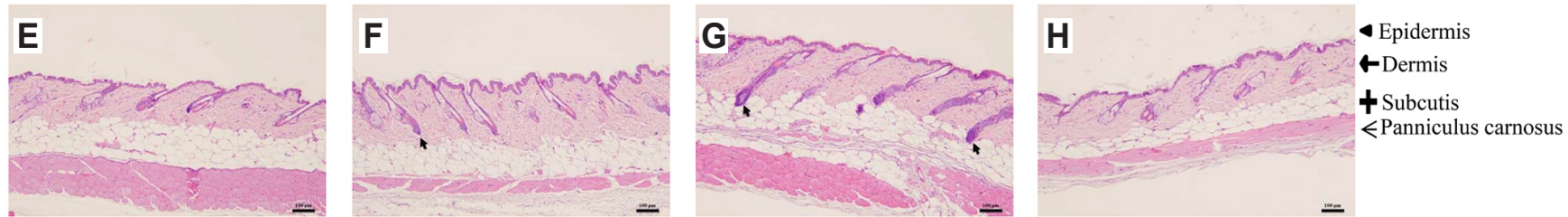

\section{2 weeks}
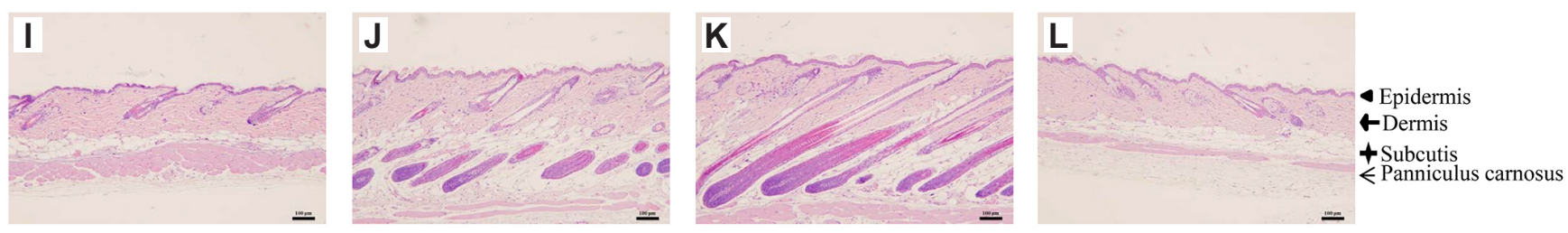

M

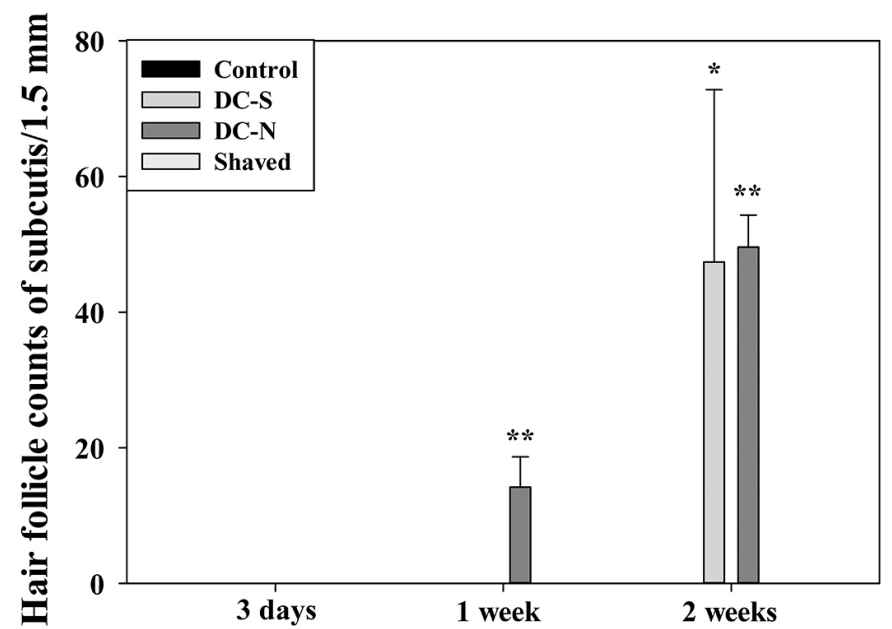

Fig. 2. Induced anagen entry and number of hair follicle by treatment of depilatory creams. Representative H\&E stain (10X) of longitudinal sections of dorsal skin tissues received topical application of depilatory creams and shaved and control (untouched) treatments for three days (A-D), one week $(E-H)$ and two weeks $(I-L)$ in BALB/c mice $(n=5)$. (M) The number of hair follicles in subcutis were counted and presented as mean $\pm S D$ and analyzed by one-way RMANOVA with a post-hoc analysis of Holm Sidak test for pairwise comparisons. Scale bar, $100 \mu \mathrm{m}$. Arrow, hair follicle in the early anagen. ${ }^{*} p<0.05$ and ${ }^{* *} p<0.01$ vs. control. 
irritation or dermatitis on the treated skin area. Fig. 1A-L shows the representatives of the H\&E stain of the transverse skin sections with different treatments from the same animal. One of the apparent histological changes observed under morphological examination was the thickness of different layers of the skin. The thickness of the epidermal layer was increased to $\sim 1.8$ to 2 folds of control by depilatory creams at three days, and was gradually returned to a level comparable to the control two weeks after the application (Fig. 1M). The width of the dermal layer was reduced in the DC-N treated skin sample at three days and DC-S-treated sample at one week, and became substantially greater than the control at two weeks (Fig. 1N). Surprisingly shaving process showed a similar trend but to a lesser extent of morphological changes in the dermis as depilatory creams (Fig. 1N). As for the changes in the subcutis, although the thickness of the hypodermis was initially decreased in the three-day samples treated with depilatory creams, it was robustly increased to $\sim 4$ folds of control in the two-week skin sections because it was packed with HFs (Fig. 1J, K, O).

\section{Effects of depilatory creams on HF development in mouse}

The morphological observation of the skin sections collected one and two weeks after the treatment of depilatory creams showed a significant increase in the number of HFs (Fig. 1) which has been considered as an indicator for the transition of hair growth from the telogen to the anagen phases [10]. The anagen phase HF has bigger follicle size and lies in deep subcutis when compared to the telogen phase HF which lies in the dermis only [10]. As shown in the representative longitudinal sections, all of the HFs of all groups were in the telogen stage at day three (Fig. 2A-D). One week after the application of DC-N, some of the HFs were apparently in the anagen phase according to the Chase's protocol [11] (Fig. 2G, arrows), whereas the majority in the control or shaved groups remained in the telogen stage. Some of the HFs in the DC-S-treated skin were in the early anagen phase at this time point (Figs. 1F, 2F, arrows). Two weeks after the application of depilatory creams, the HFs in the anagen phase were extended deep into the subcutis (Fig. 2J, K), while the control or shaved groups showed no anagen induction. These observations implied that depilatory creams induced the progression of hair cycle of HFs. As shown in Fig. 2M, the number of HFs in the subcutis was significantly increased in the DC-N treated skin at one week comparing to the other three groups, and was greatly augmented in the DC-N and DC-S skin sections at two weeks, while the control and shaved groups showed none.

\section{Induced expression of IL- 6 and TNFs in dermal fibroblasts by depilatory creams}

IHC analysis of IL-6: In order to find out which factor might be involved in the morphological changes caused by depilatory creams, IHC staining was conducted with antisera against proinflammatory factor IL-6, TNF- $\alpha$, and TNF- $\beta$ because there was a thickening in the epidermal layer three days after the application of depilatory creams. The IHC results showed that topical application of depilatory creams increased significantly the number of the IL-6 expressing dermal fibroblast (Fig. 3B, C, arrows) with a percentage of $47 \%$ and $42 \%$ for DC-S and DC-N respectively as compared to $14 \%$ and $28 \%$ for control and shaved groups respectively at day three (Fig. 3M). One week after the treatments, there were no significant difference in the percentage of IL-6 positive fibroblast among four groups, and so were the samples obtained at two weeks (Fig. 3M).

IHC analysis of TNFs: As for the analysis of TNFs, the IHC results of TNF- $\alpha$ revealed an increased population of dermal fibroblast expressed TNF- $\alpha$ in the DC-N-treated skin sections at all three time points (Fig. 4C, G, K, arrows) with a percentage of $\sim 40 \%$ at early time points and drop to $\sim 20 \%$ at two weeks (Fig. $4 \mathrm{M})$. DC-S also induced the expression of TNF- $\alpha$ in the dermal fibroblast at three days, but not at one and two weeks (Figs. 4B, F, $\mathrm{J}, 4 \mathrm{M})$. The shaving process did not affect the expression significantly different from that of control (Fig. 4M). The intensity of the staining with TNF- $\beta$ antibody was convincingly stronger in the epidermal layer of the skins treated with depilatory creams at three days, an observation that was not found one week after (Fig. $5 \mathrm{~B}, \mathrm{C})$. Close examinations showed that a significantly higher population of the dermal fibroblasts expressed TNF- $\beta$ in the depilatory cream-treated samples as compared to the control and shaved groups at three days, with a percentage of $47 \%$ and $36 \%$ for DC-S and DC-N respectively (Fig. 5M). The difference in the expression percentage of TNF- $\beta$ positive fibroblast was not found in the samples collected one and two weeks after the treatments.

\section{DISSCUSSION}

Except for the purpose of removing hair, depilatory agents have been considered for the application of enhancing transdermal penetration of drugs. Chemical depilatories usually contain 5\%$6 \%$ calcium thioglycolate and maintain a $\mathrm{pH}$ value of approximately 12 . Proper use of depilatory creams is generally considered harmless to the skin and does not influence the speed of growth or morphology of hair. However, consumers and internet users often wonder if hair grows back thicker or more in quantity after they use depilatory products. Concerning the principle employed in hair-removal products is by breaking down the external hair's keratin, it is almost unlikely to leave the skin unaffected while removing hair, since skin also contains keratin in the outer layers (corneum). Even though Duit et al. [7] reported that thioglycolate induced a reversible damage to the cornified envelopes isolated from the stratum corneum of the human epidermis within $48 \mathrm{~h}$, a thorough examination of depilatory agents' effects on the skin 


\section{Control}

\section{3 days}

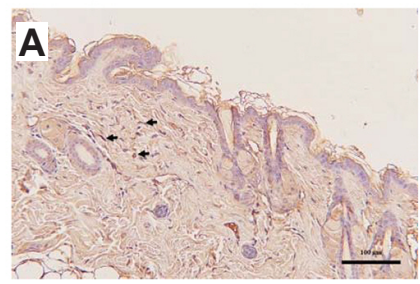

1 week

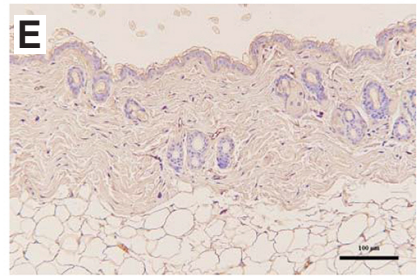

\section{2 weeks}
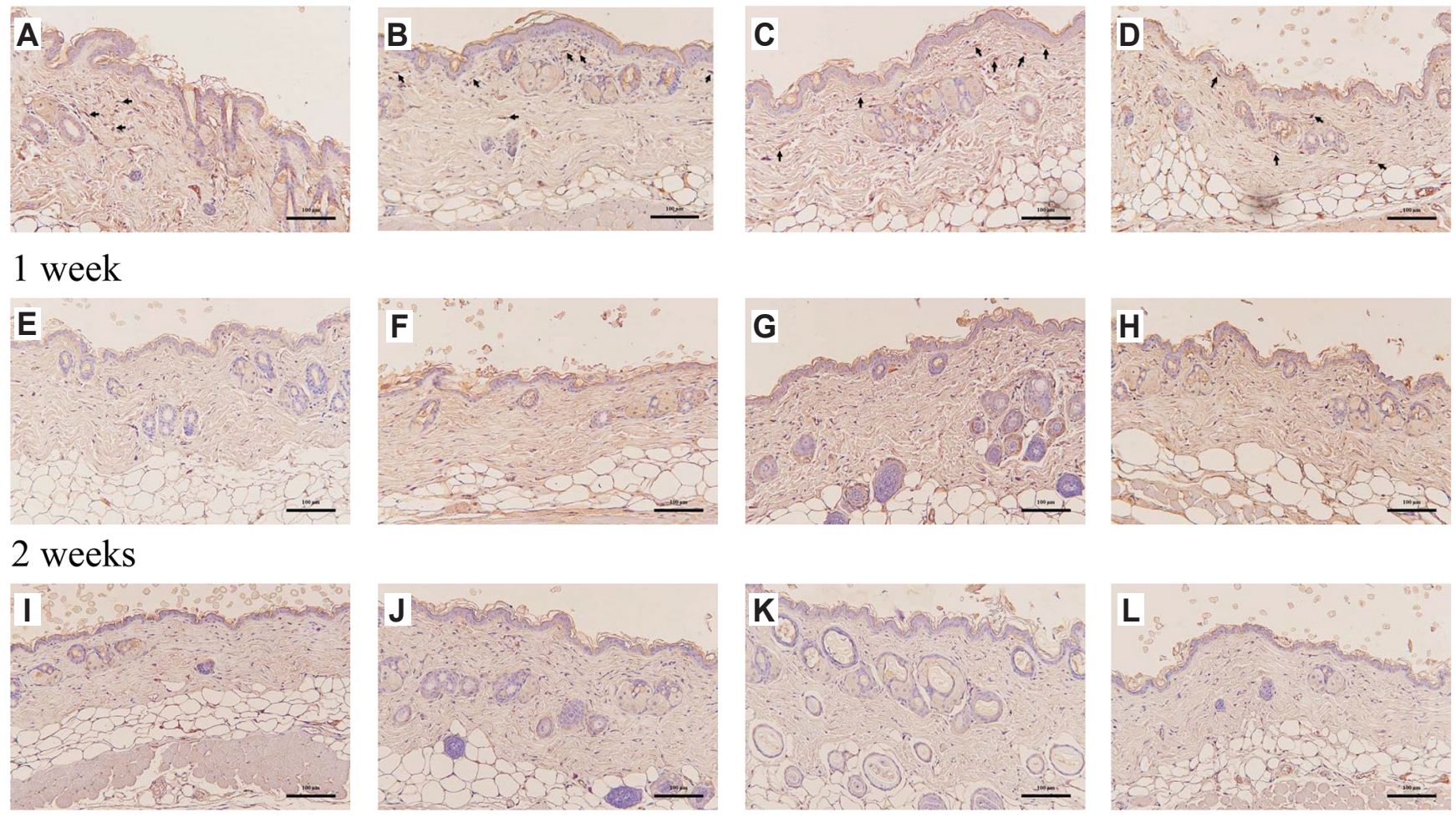

M
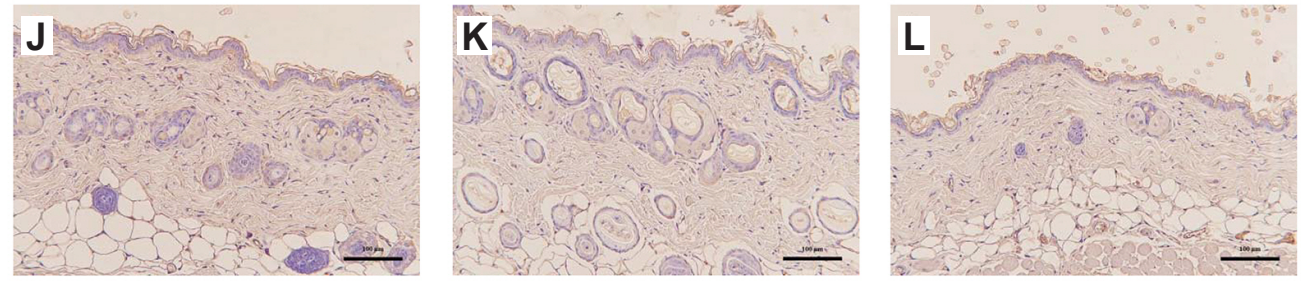

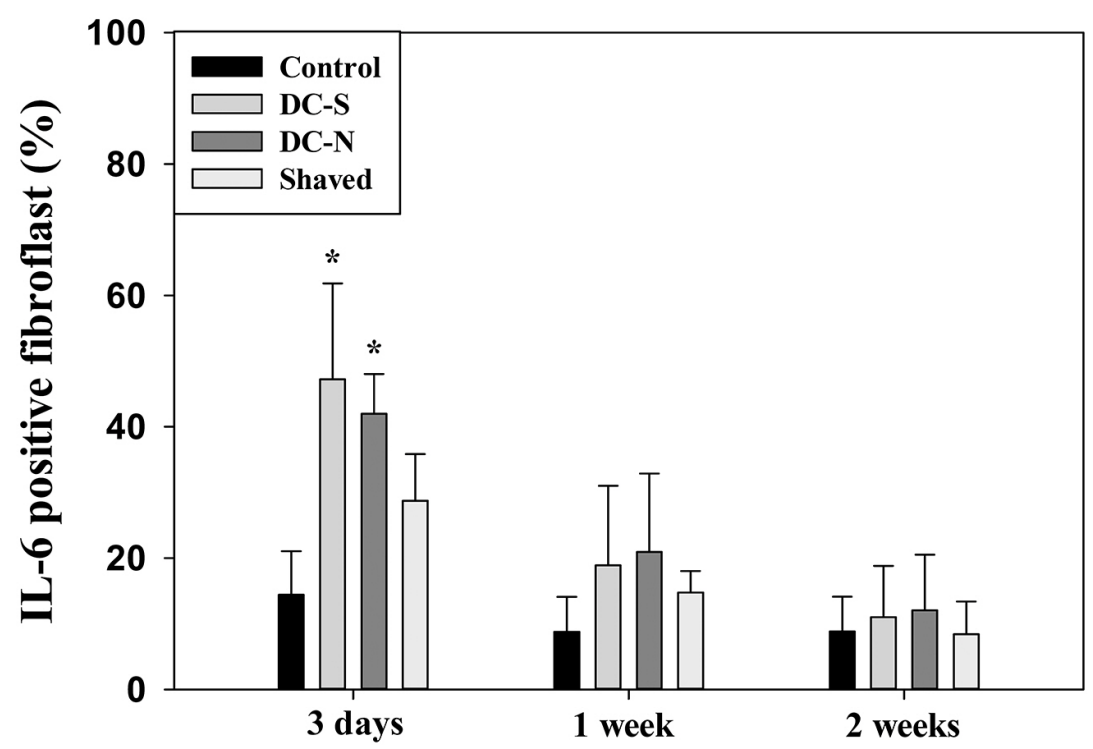

Fig. 3. Induced expression of interleukin-6 (IL-6) in dermal fibroblasts by depilatory creams. Representative of histological images (20x) stained with IL- 6 antiserum of dorsal skin tissues received topical application of depilatory creams and shaved and control (untouched) treatments for three days $(A-D)$, one week $(E-H)$ and two weeks $(I-L)$ in BALB/c mice $(n=5)$. (M) Percentage of IL-6 positive fibroblast. Scale bar, $100 \mu \mathrm{m}$. All data were presented as mean \pm SD and analyzed by one-way RMANOVA with a post-hoc analysis of Holm Sidak test for pairwise comparisons. Arrow, IL-6 positive fibroblast. ${ }^{*} p<0.05$ vs. control.

should be conducted in vivo for a longer period, especially in advance of their use as enhancers of transdermal drug delivery.

In the present study we observed the effects of depilatory creams on the skin at three different time points representing the acute and long-term responses. Shaving that physically cuts the external part of the hair was also used as a control to discriminate 
Control

3 days

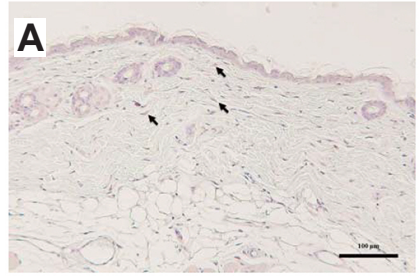

\section{1 week}

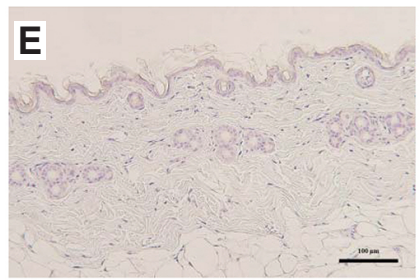

\section{2 weeks}

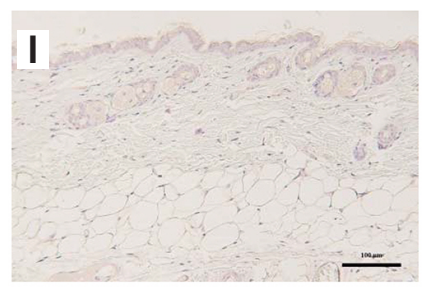

DC-S
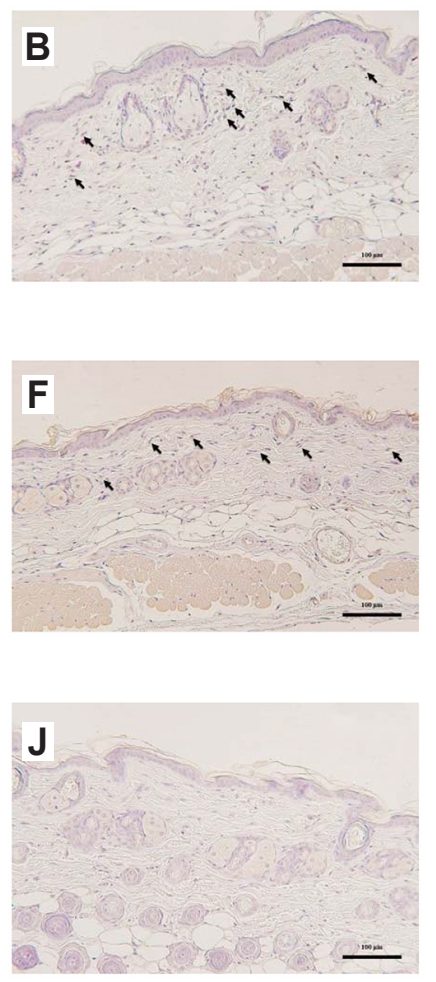

DC-N
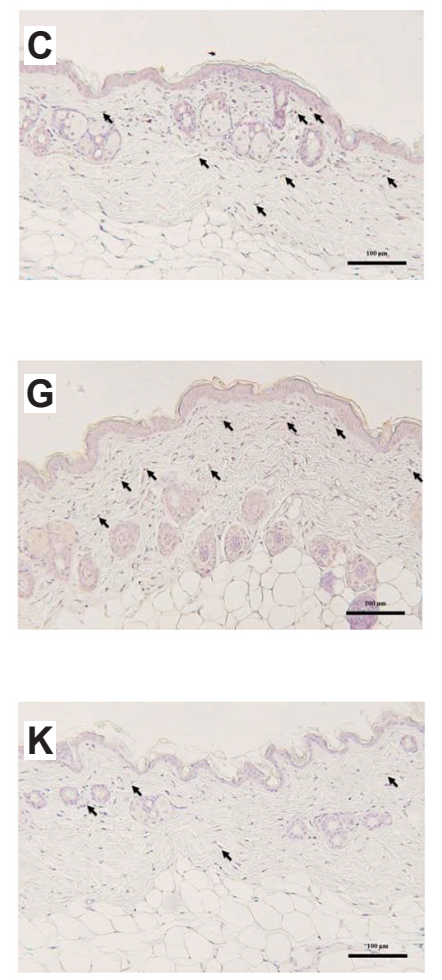

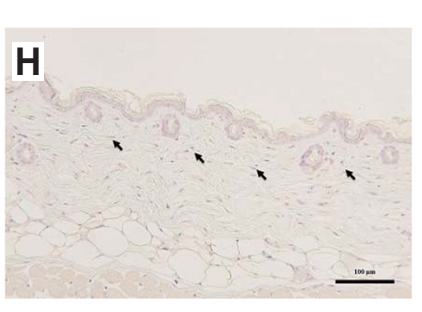

\section{Shaved}
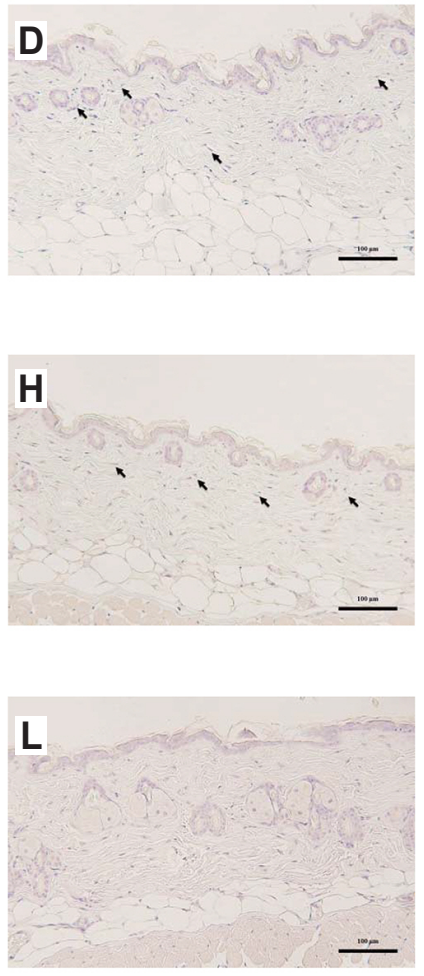

M

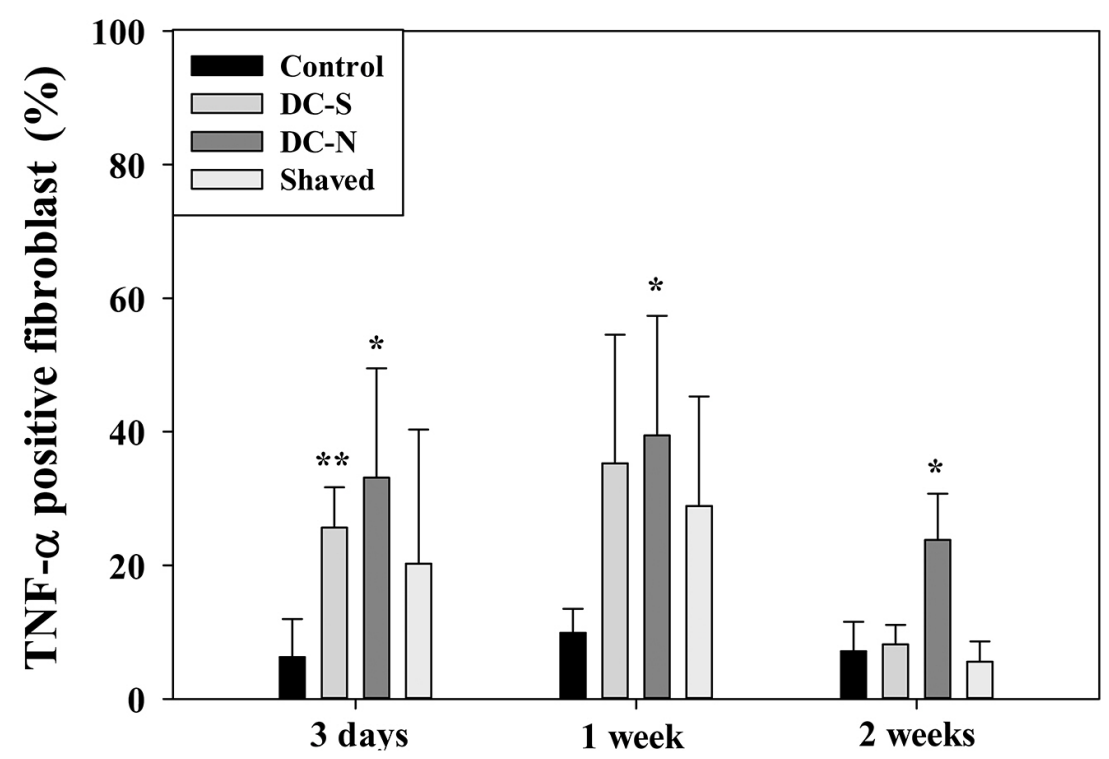

Fig. 4. Induced expression of tumor necrosis factor- $\alpha$ (TNF- $\alpha$ ) in dermal fibroblasts by depilatory creams. Representative of histological images (20x) stained with TNF- $\alpha$ antiserum of dorsal skin tissues received topical application of depilatory creams and shaved and control (untouched) treatments for three days (A-D), one week $(E-H)$ and two weeks $(I-L)$ in BALB/c mice $(n=5)$. (M) Percentages of TNF- $\alpha$ positive fibroblast. Scale bar, 100 $\mu \mathrm{m}$. All data were presented as mean \pm SD and analyzed by one-way RMANOVA with a post-hoc analysis of Holm Sidak test for pairwise comparisons. Arrow, TNF- $\alpha$ positive fibroblast. ${ }^{*} p<0.05$ and ${ }^{* *} p<0.01$ vs. control.

the effects of chemical breakdown of external hair by depilatory creams. Unlike the control skin which had HFs in the telogen phase at all three time points, depilatory creams revealed a hair growth-promoting activity as evidenced by the increased HF numbers in the subcutis at 1 and 2 weeks. The thickening of the epidermal layer 3 days after treatment with the depilatory cream 
Control

3 days

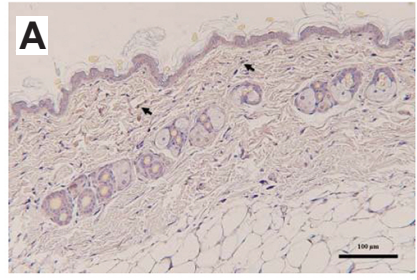

\section{1 week}

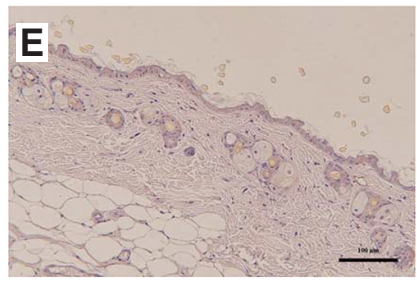

2 weeks

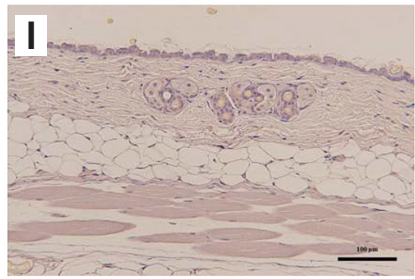

DC-S
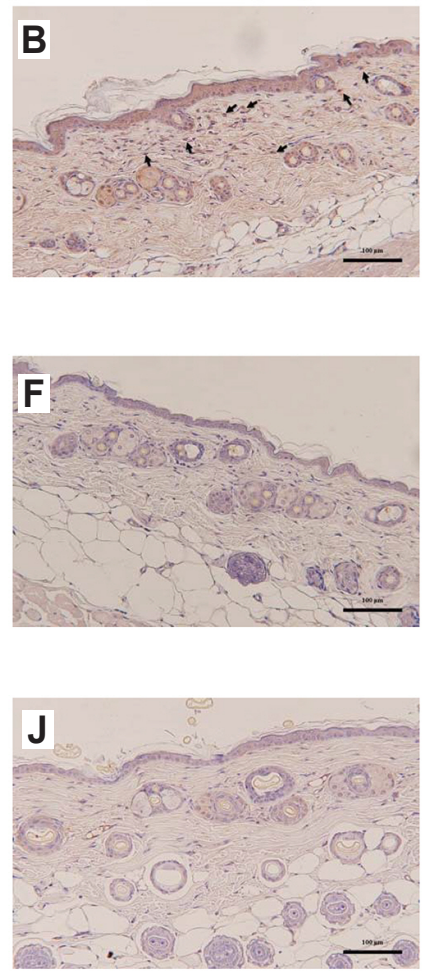

DC-N
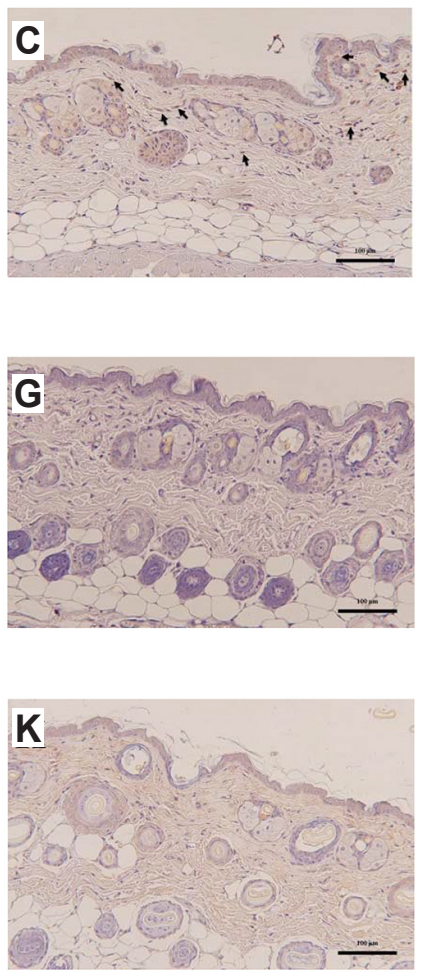

Shaved
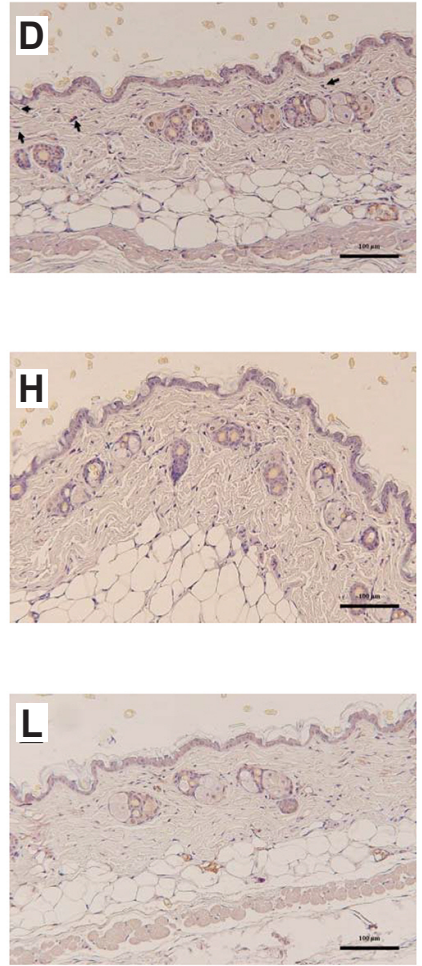

M

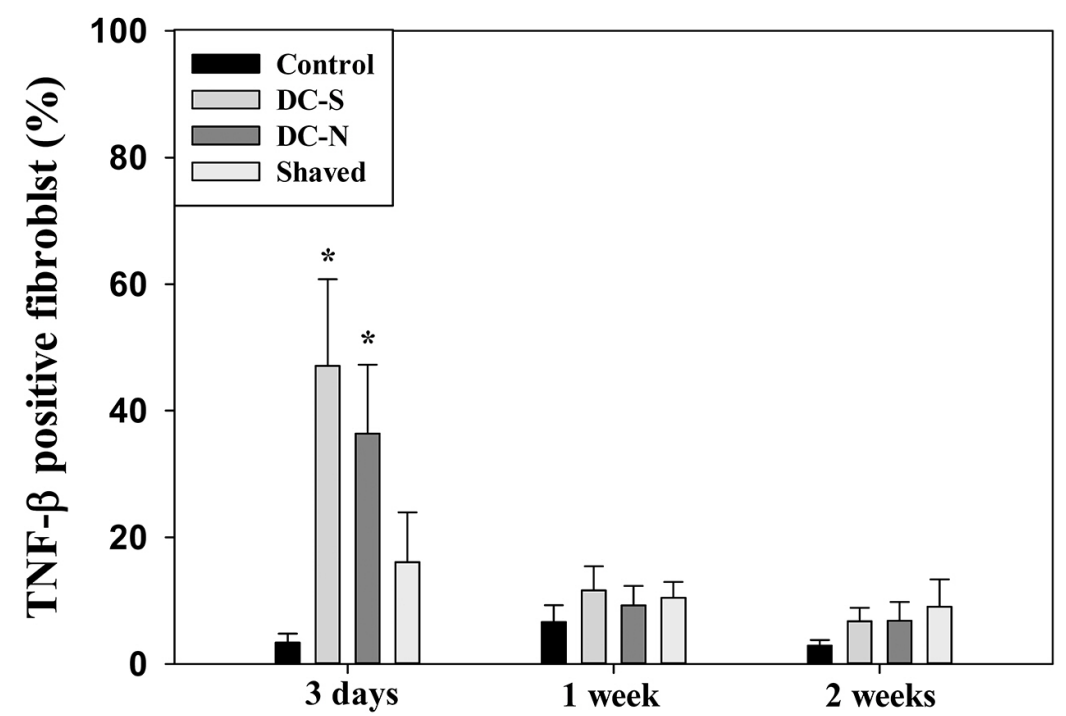

Fig. 5. Induced expression of tumor necrosis factor- $\beta$ (TNF- $\beta$ ) in dermal fibroblasts by depilatory creams. Representative of histological images (20X) stained with TNF- $\beta$ antiserum of dorsal skin tissues received topical application of depilatory creams and shaved and control (untouched) treatments for three days (A-D), one week $(E-H)$ and two weeks $(I-L)$ in BALB/c mice $(n=5)$. (M) Percentage of TNF- $\beta$ positive fibroblast. Scale bar, $100 \mu$ m. All data were presented as mean \pm SD and analyzed by one-way RMANOVA with a post-hoc analysis of Holm Sidak test for pairwise comparisons. Arrow, TNF- $\beta$ positive fibroblast. ${ }^{*} p<0.05$ vs. control.

indicated that depilatory creams may cause an inflammatory response possibly related to the increase in hair growth rate. We also observed more dermal fibroblasts expressing IL-6 and TNFs in the skin samples treated with the depilatory cream, which supports this conjecture. This inflammatory response lasted for a week, since the thickening of the epidermal layer and the increased percentages of IL- 6 and TNF- $\beta$ positive fibroblasts were diminished at one week, although TNF- $\alpha$ was remained high, 
a time point that showed a higher number of HFs in the anagen phase in the depilatory cream-treated skin samples.

IL-6, an inflammatory cytokine, is involved in the inflammatory response that occurs after cutaneous wounding and is a major event for healing process $[12,13]$. A study by $\mathrm{Pu}$ et al. [14] demonstrated that IL- 6 from adipose-derived stem cells increased cell proliferation and the number of HFs in a skin flap model. An assessment of tissue responses in rodent skin using a nonablative fractional laser (at a beam density of $1048 \mathrm{MTZ} / \mathrm{cm}^{2}$ ) revealed that moderate increases in IL-6, TNF- $\alpha$, and IL-1 $\beta$ were associated with anagen induction during HF regeneration under transient moderate inflammation in a period of 5 days [15]. Intense prolonged inflammation, however, preceded ulcer formation. Our results agreed with these reports, suggesting that depilatory creams caused minor inflammation, and that IL-6 may have mediated the induction of HF growth.

Researches have shown that the induced of TNF- $\alpha$ expression contributes to the development and growth of HF [15-18]. Anagen induction of $\mathrm{HF}$ regeneration during transient moderate inflammation by a nonablative fractional laser resulted in increased levels of TNF- $\alpha$ [15]. Transforming growth factor- $\beta$ activated kinase 1 , an inflammatory mediator required for anagen induction and maintenance of mature hair, acted through TNF- $\alpha$ to activate NF- $\kappa \mathrm{B}$ [17]. TNF- $\alpha$ also accelerated wound healing through paracrine mechanisms that are essential to controlling the hair cycle [18]. An early study suggested that TNF- $\beta$ is expressed during catagen and might be involved in follicular apoptosis [19]. To our knowledge, no other report has linked TNF- $\beta$ to the development of the hair cycle.

The two brands of depilatory creams used in this study had varying effects on the expression of TNFs. DC-N, but not DC-S, had a persistent effect on the expression of TNF- $\alpha$, but not on that of TNF- $\beta$, resulting in higher levels in the 2-week sample than in the other three samples (Fig. 4). This result coincided with the observation that DC-N presented an earlier hair growth promoting effect than did DC-S (Figs. 1, 2); although the end results for both depilatory creams were the same. The role of TNF- $\beta$ in this study must be clarified further to determine whether it functions as an integral mediator, or whether the observed effects were merely an inflammatory response unrelated to the hair growth-promoting properties of depilatory creams.

The exact molecular mechanisms responsible for depilatory cream induced anagen entry were unclear. Prior experiments have demonstrated that inflammatory cells may be involved in open wound-induced anagen entry [20], and that inflammation caused by dermatitis also induces anagen entry [21]. These observations, along with the result of our study, suggest that inflammation may be a common pathway leading to anagen entry. The rationale that transient minor inflammation promotes hair growth is further supported by the skin responses to shaving process that increased the thickness of epidermis and dermis as depilatory creams did, but did neither induce the expression of proinflammatory cytokines, IL-6 and TNFs, in the dermal fibroblasts nor the number of hair follicle. The results of Wu et al. [15] also revealed that a very low level of inflammation was unable to induce premature anagen entry. Although Seo et al. [22] reported that Hydrogen peroxide and monoethanolamine, key ingredients of hair dye, induced dermatitis and hair loss, the inflammatory response in the 3 days study was more severe than those observed in our study, suggesting that intense inflammation may cause skin damage and hair loss. In addition, the mouse model used in this study may not reflect conditions in humans because the follicular density of mouse skin is much higher than that of human skin.

The results of this study provide useful information for adjusting the composition of active ingredients of depilatory cream to minor the inflammatory response of the skin to avoid inducing hair growth and intervening with transdermal drug delivery.

\section{ACKNOWLEDGEMENTS}

The authors would like to thank Chang Gung University of Science and Technology and Chung Shan Medical University for providing the necessary facilities.

\section{CONFLICTS OF INTEREST}

The authors declare no conflicts of interest.

\section{REFERENCES}

1. Clausen T, Schwan-Jonczyk A, Lang G, Schuh W, Liebscher KD, Springob C, Franzke M, Balzer W, Imhoff S, Maresch G, Bimczok R. Hair preparations. In: Bellussi G, Bohnet M, Bus J, Drauz K, Greim H, Jackel KP, Karst U, Kleemann A, Kreysa G, Laird T, Meier W, Ottow E, Roper M, Scholtz J, Sundmacher K, Ulber R, Wietelmann U, editors. Ullmann's encyclopedia of industrial chemistry. 7th ed. Weinheim: Wiley-VCH Verlag GmbH \& Co. KGaA.; 2006. p.204241.

2. Pohl S, Varco J, Wallace P, Wolfram LJ. Hair preparations. In Othmer K, editor. Kirk-Othmer chemical technology of cosmetics. 5th ed. Hoboken (NJ): John Wiley \& Sons; 2013. p.85-122.

3. Kushida K, Masaki K, Matsumura M, Ohshima T, Yoshikawa H, Takada K, Muranishi S. Application of calcium thioglycolate to improve transdermal delivery of theophylline in rats. Chem Pharm Bull (Tokyo). 1984;32:268-274.

4. Zakzewski CA, Wasilewski J, Cawley P, Ford W. Transdermal delivery of regular insulin to chronic diabetic rats: effect of skin preparation and electrical enhancement. J Control Release. 1998;50:267-272.

5. Kanikkannan N, Singh J, Ramarao P. Transdermal iontophoretic delivery of bovine insulin and monomeric human insulin analogue. J Control Release. 1999;59:99-105.

6. Lee JN, Jee SH, Chan CC, Lo W, Dong CY, Lin SJ. The effects of 
depilatory agents as penetration enhancers on human stratum corneum structures. J Invest Dermatol. 2008;128:2240-2247.

7. Duit R, Hawkins TJ, Määttä A. Depilatory chemical thioglycolate affects hair cuticle and cortex, degrades epidermal cornified envelopes and induces proliferation and differentiation responses in keratinocytes. Exp Dermatol. 2019;28:76-79.

8. Paus R, Maurer M, Slominski A, Czarnetzki BM. Mast cell involvement in murine hair growth. Dev Biol. 1994;163:230-240.

9. Chase HB, Eaton GJ. The growth of hair follicles in waves. Ann N Y Acad Sci. 1959;83:365-368.

10. Datta K, Singh AT, Mukherjee A, Bhat B, Ramesh B, Burman AC. Eclipta alba extract with potential for hair growth promoting activity. J Ethnopharmacol. 2009;124:450-456.

11. Chase HB. Growth of the hair. Physiol Rev. 1954;34:113-126.

12. Gallucci RM, Simeonova PP, Matheson JM, Kommineni C, Guriel JL, Sugawara T, Luster MI. Impaired cutaneous wound healing in interleukin-6-deficient and immunosuppressed mice. FASEB J. 2000;14:2525-2531.

13. Lin ZQ, Kondo T, Ishida Y, Takayasu T, Mukaida N. Essential involvement of IL- 6 in the skin wound-healing process as evidenced by delayed wound healing in IL-6-deficient mice. J Leukoc Biol. 2003;73:713-721.

14. Pu CM, Chen YC, Chen YC, Lee TL, Peng YS, Chen SH, Yen YH, Chien CL, Hsieh JH, Chen YL. Interleukin-6 from adipose-derived stem cells promotes tissue repair by the increase of cell proliferation and hair follicles in ischemia/reperfusion-treated skin flaps. Mediators Inflamm. 2019;2019:2343867.

15. Wu YF, Wang SH, Wu PS, Fan SM, Chiu HY, Tsai TH, Lin SJ. Enhancing hair follicle regeneration by nonablative fractional laser: assessment of irradiation parameters and tissue response. Lasers Surg
Med. 2015;47:331-341.

16. Philpott MP, Sanders DA, Bowen J, Kealey T. Effects of interleukins, colony-stimulating factor and tumour necrosis factor on human hair follicle growth in vitro: a possible role for interleukin-1 and tumour necrosis factor-alpha in alopecia areata. Br J Dermatol. 1996; 135:942-948.

17. Sayama K, Kajiya K, Sugawara K, Sato S, Hirakawa S, Shirakata Y, Hanakawa Y, Dai X, Ishimatsu-Tsuji Y, Metzger D, Chambon P, Akira S, Paus R, Kishimoto J, Hashimoto K. Inflammatory mediator TAK1 regulates hair follicle morphogenesis and anagen induction shown by using keratinocyte-specific TAK1-deficient mice. PLoS One. 2010;5:e11275.

18. Heo SC, Jeon ES, Lee IH, Kim HS, Kim MB, Kim JH. Tumor necrosis factor- $\alpha$-activated human adipose tissue-derived mesenchymal stem cells accelerate cutaneous wound healing through paracrine mechanisms. J Invest Dermatol. 2011;131:1559-1567.

19. Seiberg M, Marthinuss J, Stenn KS. Changes in expression of apoptosis-associated genes in skin mark early catagen. J Invest Dermatol. 1995;104:78-82.

20. Osaka N, Takahashi T, Murakami S, Matsuzawa A, Noguchi T, Fujiwara T, Aburatani H, Moriyama K, Takeda K, Ichijo H. ASK1dependent recruitment and activation of macrophages induce hair growth in skin wounds. J Cell Biol. 2007;176:903-909.

21. Kluger N, Garat H. Transient localized hypertrichosis on a temporary henna tattoo. Contact Dermatitis. 2010;62:188-189.

22. Seo JA, Bae IH, Jang WH, Kim JH, Bak SY, Han SH, Park YH, Lim KM. Hydrogen peroxide and monoethanolamine are the key causative ingredients for hair dye-induced dermatitis and hair loss. $J$ Dermatol Sci. 2012;66:12-19. 